\title{
Generalization of Magnetostatic Method of Moments for Thin Layers with Regular Rectangular Grids
}

\author{
R. SZEWCZYK*
}

Industrial Research Institute for Automation and Measurements, Al. Jerozolimskie 202, 02-486 Warsaw, Poland

\begin{abstract}
Possibilities of the modeling of the flux density distribution in thin films are significantly limited using the finite elements method due to the fast increase of the number of tetrahedral elementary cells with reduction of the thickness. For this reason, method of the moments is very important alternative for finite elements method in the case of thin layers, where layer's thickness should be considered. Method of the moments overcomes this barrier, due to the possibility of operation on uniform grids with limited number of cells. Moreover, in opposite to the finite elements method, the method of the moments requires solving of the well defined linear equations, instead of the set of ill-posed differential equations. Paper presents the generalization of the method of the moments for thin layers with given thickness. Layers are defined as the 2D rectangular grids. Within the generalization, four key equations describing the influence of rectangular cell's border on the magnetization of cells are stated. On the base of these dependences, the set of $2 N M$ linear equations was determined, where $N$ and $M$ are the numbers of rectangular cells in the rows and columns of regular grid. Finally, the set of linear equations is solved and magnetic flux density distribution in the thin layer is calculated.
\end{abstract}

DOI: 10.12693/APhysPolA.131.845

PACS/topics: 75.70.Ak, 75.50.Kj

\section{Introduction}

Modeling techniques play the key role in the process of development of modern sensors with cores made of magnetic materials. Recently, methods of modeling of sensors are dominated by the finite elements methods. However, these methods, using the tetrahedral cells, have limited usability in the case of thin films with defined thickness.

Rough estimation of number of tetrahedral cells $n_{t c}$, in square-shaped thin film with length of edge equal to $a$ and thickness $g$ is given by the following equation:

$$
n_{t c}=1.2\left(\frac{a}{g}\right)^{2} \text {. }
$$

This means that for the typical thin layer, with edge length equal to $10 \mathrm{~mm}$ and thickness equal to $20 \mu \mathrm{m}$, finite elements method require 300000 nearly-regular tetrahedral elements. Number of these elements may be limited by the adaptive meshing using e.g. the Delaunay method [1]. However, even in the case of adaptive meshing, number of elements still creates significant problems in the case of the finite elements method. Moreover, finite elements method requires solving of the set of ill-posed differential equations, which additionally consumes time and computer resources [2].

These drawbacks cause the necessity of searching for novel methods of determination of flux density distribution during the magnetization of the thin layers. One of such methods is the method of the moments [3]. However, previously presented applications of the method of

*e-mail: rszewczyk@onet.pl the moments are oriented on the specific shapes of magnetized cores $[4,5]$. For wider application of this method in development of sensors utilizing magnetic thin layers, general form of equations stating the method of the moments is required. Such equations were never presented before. This paper is filling this gap, enabling modeling any sensor utilizing the thin layer.

\section{Principles of the method of the moments}

The calculations in the method of the moments are carried out on the base of assumption that magnetic moment $m_{i}$ may be assigned to the each border of element of the magnetized body. This magnetic moment acts in opposite direction to the magnetizing field $H$, causing the demagnetization of the body. This leads to the following equation describing the magnetization of the thin, rod-shaped body [6]:

$$
M_{k}+(\mu-1) \sum_{i=0}^{n} H_{d}(i k)=(\mu-1) H_{e x t},
$$

where $\mu$ is relative magnetic permeability of the body and $H_{d}(i, k)$ is demagnetization caused by $i$-th border of rod's element, influencing on barycenter of $k$ element.

As a result, the magnetization $M_{k}$ of the $k$ element of the thin rod-shaped body, is given as

$$
\begin{aligned}
& M_{k}=(\mu-1)\left[H-\sum_{i=0}^{n} H_{d}(i, k)\right], \\
& M_{k}=(\mu-1)\left(H_{e x t}-\sum_{i=0}^{n} H_{d}(i, k)\right)
\end{aligned}
$$

Surface magnetic charge density $\sigma_{i}$ for $i$-th border, in the case of rod-shaped body, is given as

$$
\sigma_{i}=\Delta M n \Delta L,
$$

where $\Delta M$ is the difference of magnetization assigned to 
the border of elements, $\Delta L$ is the length of the element of thin rod-shaped body and $n$ is the unit vector describing the direction. Magnetic moment $m_{i}$ assigned to the $i$-th border may be easily calculated as

$$
m_{i}=\sigma_{i} s \text {, }
$$

where $s$ is the field of the border of the element. Moreover, the demagnetizing field from the magnetic moment $m_{i}$ acting on the barycenter of $k$ element is given as

$$
H_{d}\left(r_{x}\right)=\frac{1}{4 \pi}\left(\frac{3 r_{x}\left(m_{x} r_{x}\right)}{r_{x}^{5}}-\frac{m_{x}}{r_{x}^{3}}\right)=\frac{m_{i}}{2 \pi\left|r_{x}\right|^{3}},
$$

where $r_{x}$ is the distance between the barycenter of $k$ element and the border $i$. After recalculation, considering the geometry [6]:

$$
H_{d}(i, k)=\frac{\left(M_{i}-M_{i+1}\right) \Delta L s \operatorname{sgn}\left(i-k+\frac{1}{2}\right)}{2 \pi\left|\Delta L\left(i-k+\frac{1}{2}\right)\right|^{3}} .
$$

On the base of Eqs. (3) and (7), the set of $k$ linear equations may be stated. This set of equations may be easily solved, giving the distribution of magnetization in the thin rod-shaped body.

\section{Generalization of the method of the moments}

Presented idea of the method of the moments may be generalized for thin layers. Geometry of such system with uniform rectangle elements is presented in Fig. 1.

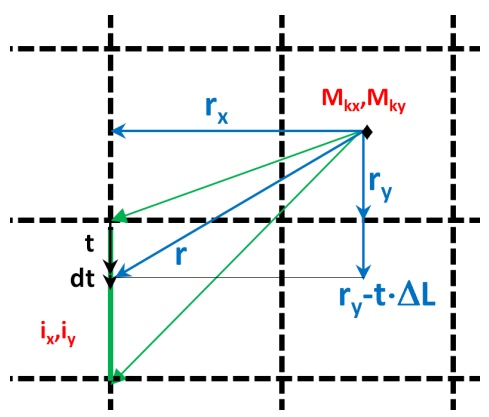

Fig. 1. Uniform rectangle elements describing the thin layer. Vertical border is marked green.

In the case of thin layer bodies, magnetization of $\left(k_{x}\right.$ $k_{y}$ ) element is given by two magnetizations: $M_{k x}, M_{k y}$. In the presented case, the following interactions should be considered:

- demagnetization $H_{d}$ caused by the horizontal border (in $x$ direction) act on the $M_{k x}$ component of the magnetization of the element,

- demagnetization $H_{d}$ caused by the horizontal border (in $x$ direction) act on the $M_{k y}$ component of the magnetization of the element,

- demagnetization $H_{d}$ caused by the vertical border (in $y$ direction) act on the $M_{k x}$ component of the magnetization of the element,
- demagnetization $H_{d}$ caused by the vertical border (in $y$ direction) act on the $M_{k y}$ component of the magnetization of the element.

In the presented case, the differential influence due to the demagnetization by the moment $d m\left(i_{x}, i_{y}\right)$ on the given axis direction of $M$ should be considered. As a result, this influence should be integrated over the whole length of the border, using the parameter $t \in\langle 0,1\rangle$. The set of equations determining the value of demagnetizing field $H_{d}$ for all four cases is presented in Table I.

TABLE I

The set of equations determining the value of demagnetizing field $H_{d}$.

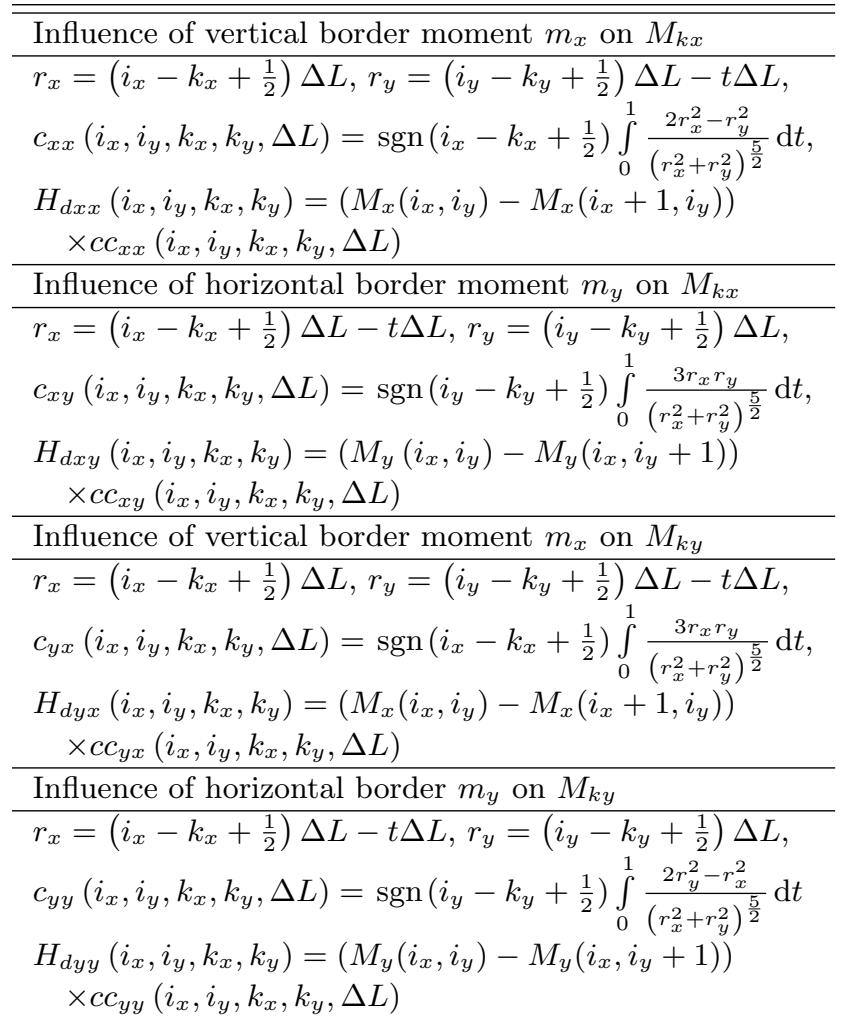

In all cases given in Table I, the parameter $c$ is

$$
c=\frac{g \Delta L^{2}}{4 \pi},
$$

where $g$ is the layer thickness, whereas $\Delta L$ is the element length.

Finally, the set of linear equations determining both $x$ and $y$ components of the magnetization $M_{k}$ is

$$
\begin{aligned}
& M_{x}\left(k_{x}, k_{y}\right) \\
& +\sum_{i y=1}^{N} \sum_{i x=0}^{M}\left[\left(\mu\left(k_{x}, k_{y}\right)-1\right) d H_{x x}\left(i_{x}, i_{y}, k_{x}, k_{y}\right)\right] \\
& +\sum_{i x=1}^{n} \sum_{i y=0}^{n}\left[\left(\mu\left(k_{x}, k_{y}\right)-1\right) d H_{x y}\left(i_{x}, i_{y}, k_{x}, k_{y}\right)\right]= \\
& \quad \mu\left(k_{x} k_{y}\right) H_{x}
\end{aligned}
$$




$$
\begin{aligned}
& M_{y}\left(k_{x}, k_{y}\right) \\
& +\sum_{i x=1}^{N} \sum_{i y=0}^{M}\left[\left(\mu\left(k_{x}, k_{y}\right)-1\right) d H_{y y}\left(i_{x}, i_{y}, k_{x}, k_{y}\right)\right] \\
& +\sum_{i y=1}^{n} \sum_{i x=0}^{n}\left[\left(\mu\left(k_{x}, k_{y}\right)-1\right) d H_{y x}\left(i_{x}, i_{y}, k_{x}, k_{y}\right)\right]= \\
& \mu\left(k_{x} k_{y}\right) H_{y} .
\end{aligned}
$$

It should be stressed that for square mesh $N$ per $M$ cells, the set of $2 N M$ linear equations may be stated. This set of equations may be solved using least-squares solution or preconditioned conjugate gradient iterative method.

\section{Results of calculations}

Presented generalization of the method of the moments was implemented using open-source software OCTAVE 4.0 , which is compatible with MATLAB. The results of calculation of flux density B distribution in the two thin layers with thickness $2 \mu \mathrm{m}$ and $70 \mu \mathrm{m}$ are presented in Fig. 2.
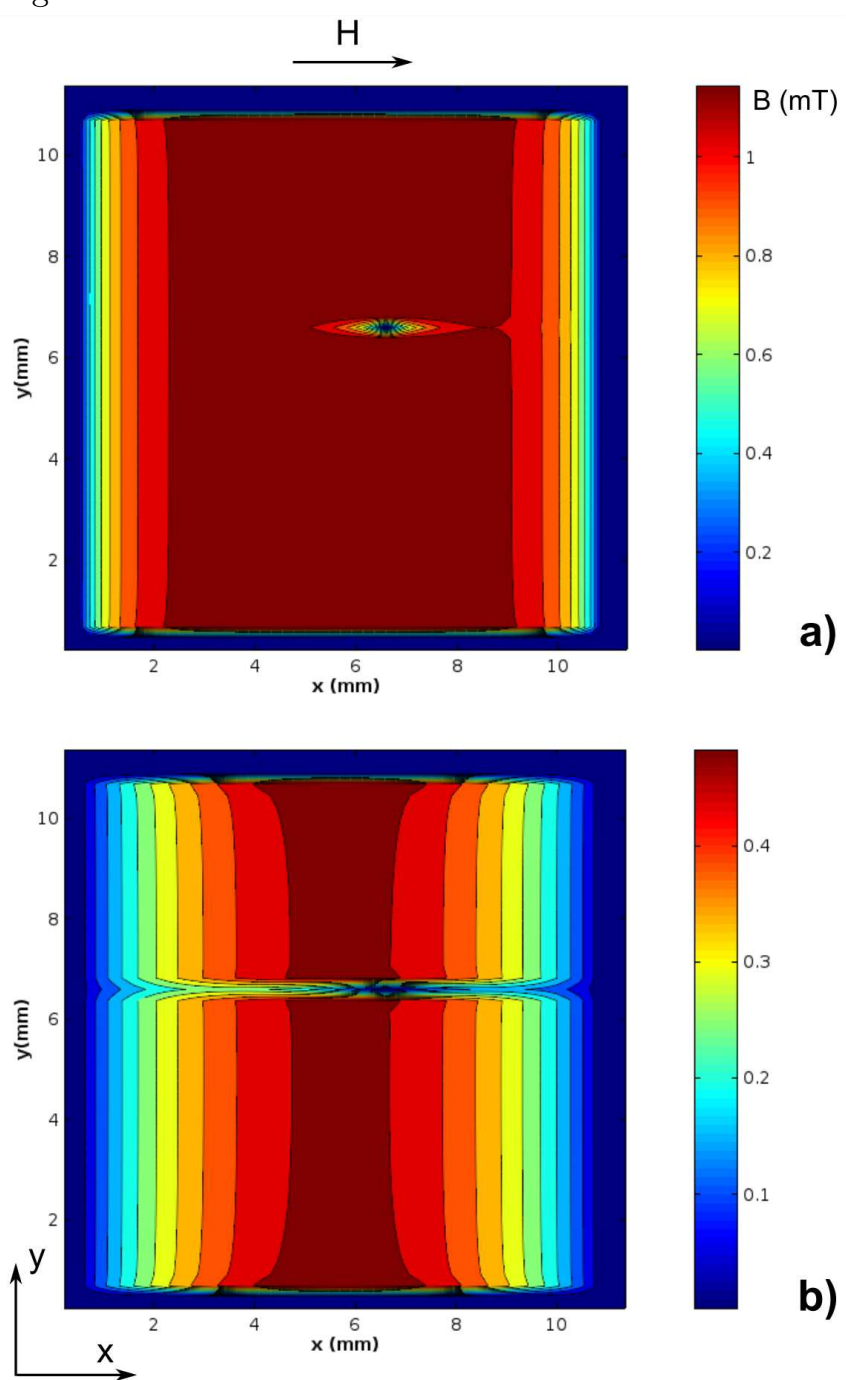

Fig. 2. Flux density $B_{x}$ distribution in thin layers with thickness: (a) $2 \mu \mathrm{m}$, (b) $70 \mu \mathrm{m}$.
Simulation was carried out for magnetizing field $H$ equal to $1000 \mathrm{~A} / \mathrm{m}$ applied in the $x$ direction. Relative permeability $\mu$ of the analyzed thin layer was 1000 , whereas its size was $10 \mathrm{~mm}$ per $10 \mathrm{~mm}$.

Presented results indicate that layer thickness has very significant influence on demagnetization of thin layer. This phenomenon should be taken into consideration during the development of sensors utilizing the thin layers as magnetic cores.

\section{Conclusions}

Generalization of the method of moments for thin layers enables fast and effective simulation of flux density distribution in thin layers. Presented generalization may be implemented in open-source software, e.g. OCTAVE. Moreover, in opposite to finite elements method, the method of moments requires only solving the well-defined linear equations.

Presented results of simulation indicate that the layer thickness has significant influence on the flux density distribution in the thin layers. This phenomena is especially important in the case of flux gate sensors utilizing cores made of amorphous alloys, which thickness vary from about $10 \mu \mathrm{m}$ up to $50 \mu \mathrm{m}$.

\section{References}

[1] J.-D. Boissonnat, M. Teillaud, Theor. Comput. Sci. 112, 339 (1993).

[2] R. Mesquita, J.P. Bastos, IEEE Trans. Magn. 28, 1044 (1992).

[3] O. Chadebec, J.-L. Coulomb, J. Fleur, IEEE Trans. Magn. 42, 515 (2006).

[4] J. Kubik, P. Ripka, Sens. Actuat. A Phys. 143, 237 (2008).

[5] H. How, L. Sun, C. Vittoria, IEEE Trans. Magn. 33, 3397 (1997).

[6] O. Chadebec, L.-L. Rouve, J.-L. Coulomb, IEEE Trans. Magn. 38, 517 (2002). 